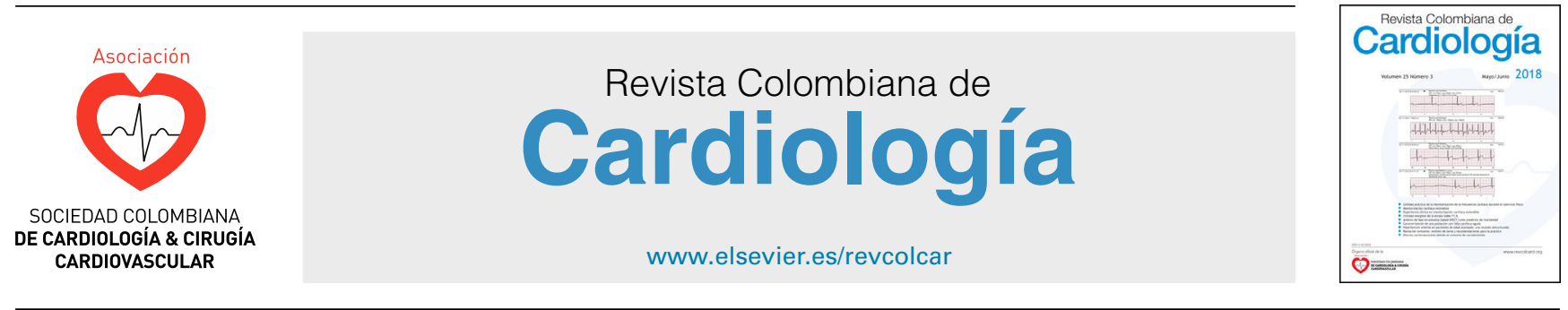

CARDIOLOGÍA DEL ADULTO - ARTÍCULO ORIGINAL

\title{
Efectos de la fragilidad en los resultados adversos de la cirugía cardiaca en ancianos
}

\section{Gustavo Alexis Lemus Barrios ${ }^{\mathrm{a}, *}$, Juan Mauricio Cárdenas Castellanos ${ }^{\mathrm{a}, \mathrm{b}}$, Carmen Lucía Curcio Borrero ${ }^{c}$ y German Alberto Moreno Gómez ${ }^{a}$}

\author{
a Grupo de investigación en Medicina Interna, Universidad Tecnológica de Pereira, Pereira, Colombia \\ b Departamento de Cardiología, Clínica Los Rosales, Pereira, Colombia \\ c Grupo de investigación en Gerontología y Geriatría, Universidad de Caldas, Manizales, Colombia
}

Recibido el 15 de mayo de 2018; aceptado el 6 de noviembre de 2018

Disponible en Internet el 10 de julio de 2019

\author{
PALABRAS CLAVE \\ Fragilidad; \\ Anciano; \\ Cirugía cardiaca
}

\begin{abstract}
Resumen
Introducción: la fragilidad es una condición del paciente anciano, caracterizada por un estado de vulnerabilidad para desenlaces adversos en salud, incluidos los causados por la cirugía cardíaca.

Objetivo: establecer la prevalencia de fragilidad en el contexto prequirúrgico del anciano sometido a cirugía cardiaca y establecer el riesgo de desenlaces adversos otorgado por esta condición.

Metodología: estudio analítico de cohorte que valoró la fragilidad en 66 pacientes ancianos sometidos a cirugía cardiaca mediante el fenotipo físico de fragilidad de Fried. Se realizó un seguimiento a 30 días, durante el cual se estableció la prevalencia de fragilidad y el riesgo otorgado por esta condición a los desenlaces adversos de la cirugía cardiaca.

Resultados: la edad de los pacientes osciló entre los 60 y los 83 años; la mediana fue de 70,5 años. La prevalencia de la fragilidad fue del $31,8 \%$. La fragilidad incrementó los siguientes desenlaces adversos postoperatorios: muerte ( $\mathrm{p} 0,001)$, ventilación prolongada ( 0,001$)$, uso prolongado de vasoactivos ( 0,001$)$ e infección del sitio operatorio $(p 0,004)$. El riesgo de mortalidad otorgado por la fragilidad fue más alto que el de los puntajes usuales de valoración como el EuroSCORE II (HR 5,49; IC95\% 1,0-28,5 vs. HR 1,17 IC95\% 1,0-1,29).

Conclusión: la fragilidad es una condición frecuente en el paciente anciano sometido a cirugía cardiaca e incrementa el riesgo de eventos adversos, en especial el de mortalidad. Se recomienda incluir la medición de la fragilidad en el protocolo prequirúgico del paciente anciano. (C) 2019 Sociedad Colombiana de Cardiología y Cirugía Cardiovascular. Publicado por Elsevier España, S.L.U. Este es un artículo Open Access bajo la licencia CC BY-NC-ND (http:// creativecommons.org/licenses/by-nc-nd/4.0/).
\end{abstract}

\footnotetext{
* Autor para correspondencia.

Correo electrónico: cardiogus@gmail.com (G.A. Lemus Barrios).
} 


\section{KEYWORDS}

Frailty;

Elderly;

Cardiac surgery
Effects of frailty on the adverse outcomes of cardiac surgery in the elderly

\begin{abstract}
Introduction: Frailty is a common condition in the elderly patient, and is noted for its vulnerability for adverse outcomes in health, including those caused by cardiac surgery.

Objective: To establish the prevalence of frailty in the pre-surgical context of the elderly subjected to cardiac surgery, as well as to establish the risk of adverse outcomes due to this condition.

Method: An analytical cohort study was performed in order to evaluate frailty in 66 elderly patients subjected to cardiac surgery, using the physical phenotype of frailty described by Fried. A follow-up was made at 30 days, during which the frailty prevalence was established, as well the risk of adverse outcomes of the cardiac surgery due to this condition.

Results: The age of the patients varied between 60 and 83 years, with a median of 70.5 years. The prevalence of frailty was $31.8 \%$. Frailty increased the following post-surgical adverse outcomes: death $(P=.001)$, longer ventilation time $(P=.001)$, prolonged use of vasoactive drugs $(P=.001)$, and infection of the surgical site $(P=.004)$. The mortality risk due to frailty was higher than that of the usual evaluation scores such as Euro SCORE II (HR 5.49; 95\% Cl; 1.0-28.5 vs. HR 1.17: $95 \% \mathrm{Cl} ; 1.0-1.29)$.

Conclusion: Frailty is a common condition in the elderly patient subjected to cardiac surgery, and increases the risk of adverse events, particularly, mortality. It is recommended to use measurements of frailty in the pre-surgical protocol of the elderly patient.

(c) 2019 Sociedad Colombiana de Cardiología y Cirugía Cardiovascular. Published by Elsevier España, S.L.U. This is an open access article under the CC BY-NC-ND license (http:// creativecommons.org/licenses/by-nc-nd/4.0/).
\end{abstract}

\section{Introducción}

Los avances en protección miocárdica, técnica quirúrgica, circulación extracorpórea y cuidados anestésicos han hecho que la cirugía cardiaca sea cada vez más segura y que se pueda hacer este tipo de procedimientos en pacientes de mayor edad ${ }^{1}$; sin embargo, la evaluación del riesgo quirúrgico en ancianos es un reto, pues no solo se debe tener en cuenta la comorbilidad, sino también otras condiciones geriátricas como la fragilidad, el estado cognitivo y la polifarmacia. Los puntajes comúnmente utilizados en la valoración prequirúrgica cardiaca como el score STS (Society of Thoracic Surgeons score: puntaje de la sociedad de cirujanos torácicos de EEUU) y EuroSCORE II (sistema europeo de puntuación de evaluación de riesgo quirúrgico cardíaco) tienen algunas limitaciones en la población anciana: primero, derivan de estudios de población, principalmente de mediana edad; así mismo, se basan en la edad cronológica mas no en la biológica, y por último no tienen en cuenta una medición completa de la fragilidad pese a que hay evidencia de que es un factor de riesgo independiente de resultados adversos postoperatorios ${ }^{3-5}$. Lo anterior genera una limitación en la predicción de los resultados de la cirugía cardiaca en los ancianos, observándose sobreestimación ${ }^{6,7}$ y subestimación ${ }^{8}$ de la mortalidad y otras complicaciones.

La fragilidad es una condición geriátrica que se define como un estado biológico caracterizado por incremento en la vulnerabilidad y disminución de la resistencia al estrés fisiológico ${ }^{9}$ y se comporta como un predictor de mortalidad independiente de la edad, la comorbilidad y la severidad de la enfermedad y de la discapacidad ${ }^{2}$. Debido a que la cirugía cardiaca es un factor estresante significativo ${ }^{10}$, la presencia de fragilidad en el paciente anciano se correlaciona con desenlaces posoperatorios adversos ${ }^{11-14}$. Tanto la Asociación Americana del Corazón (AHA) (American Heart Association) como la Sociedad Europea de Cardiología (SEC), resaltan la importancia de la valoración de la fragilidad del paciente geriátrico con enfermedad cardiovascular ${ }^{2,15}$, sin embargo, a pesar de la importancia clínica, su medición no está claramente incluida en las guías de manejo de cirugía cardiovascular, ni se describe el mejor método para evaluarla. Actualmente, existen más de 67 herramientas para llegar el diagnóstico de fragilidad ${ }^{16}$, no obstante, las dos más citadas y estandarizadas son en orden, el fenotipo físico de fragilidad (también conocido como el Índice de Fried) y el índice de acumulación de déficit, también conocido como índice de Rockwood ${ }^{17}$, éste último de mayor complejidad ya que al tener un número importante de variables (70 ítems) se hace poco práctico para el médico no geriatra. El fenotipo físico de fragilidad, por otro lado, fue desarrollado en el año 2001 por Linda Fried y su grupo ${ }^{9}$ basados en la cohorte Cardiovascular Health Study (CHS) en la que estandarizaron y validaron los criterios diagnósticos para fragilidad los cuales se basan en cinco hallazgos:

1. Pérdida de peso no intencionada.

2. Agotamiento o fatiga.

3. Actividad física.

4. Velocidad de marcha.

5. Debilidad.

Los sujetos con uno o dos criterios se consideran pre- $y$ aquéllos con 3 o más criterios se consideran frágiles. 
Otras condiciones geriátricas que están ausentes en los puntajes de riesgo usuales son la comorbilidad, la polifarmacia y el estado cognitivo. La polifarmacia es una condición médica de definición amplia y evolutiva; sin embargo, la más usada y aceptada es el empleo concomitante de cinco o más medicamentos ${ }^{18}$. La polifarmacia incrementa el potencial de interacciones farmacológicas y de reacciones adversas en los pacientes sometidos a cirugía, lo cual conduce a resultados quirúrgicos adversos en algunos estudios clínicos ${ }^{19,20}$. La comorbilidad, definida desde 1970 por Feinstein ${ }^{21}$ como la existencia de una condición médica adicional a la enfermedad indexada bajo estudio ${ }^{22}$, es también una condición común en los pacientes ancianos, cuya medición se puede realizar por múltiples escalas o índices, no obstante, el más usado en la investigación es el índice de Charlson $^{23}$, el cual puntúa una serie de comorbilidades que individualmente o en combinación pueden incidir en el riesgo de mortalidad a corto plazo de pacientes incluidos en estudios de investigación ${ }^{22}$, desde la definición inicial se considera que un paciente tiene muy alta comorbilidad y por tanto mayor mortalidad cuando el puntaje es superior a 3 puntos ${ }^{23}$. Finalmente, el estado cognitivo preoperatorio de los pacientes sometidos a cirugía no ha sido tan ampliamente estudiado como el estado cognitivo post-operatorio; en un meta-análisis de Stroobant et al.. ${ }^{24}$, solo se encontraron 8 artículos de calidad que revisaran esta condición preoperatoria en los pacientes bajo cirugía cardiaca; dado el impacto potencial que podría tener el estado cognitivo en los resultados de la cirugía cardiaca, algunos autores han fomentado la realización de estudios que valoren esta condición geriátrica ${ }^{24}$. Pese a que no hay una herramienta estándar para la medición del estado cognitivo en pacientes sometidos a cirugía cardiaca, el Mini Mental test (Mini Mental State Examination- MMSE) es una herramienta de tamización ampliamente estandarizada y validada en territorio colombiano para esta condición siempre y cuando sea ajustada al nivel educativo del sujeto evaluado ${ }^{25}$.

Esta investigación estuvo orientada a la valoración de fragilidad mediante el índice de fragilidad de Fried ${ }^{9}$ en población anciana que fue llevada a cirugía cardiovascular en un centro de referencia de la ciudad de Pereira y evaluó el riesgo de la fragilidad con resultados post operatorios adversos. Teniendo en cuenta que los pacientes ancianos tienen otras condiciones geriátricas con potencial de desenlaces quirúrgicos adversos, también se midió la polifarmacia, la comorbilidad y el estado cognitivo. En la literatura revisada no se encontraron estudios en Colombia que evaluaran la fragilidad en ancianos llevados a cirugía cardiovascular. El conocimiento sobre este tema puede servir como una guía para toma de decisiones clínicas y como objeto de investigación e intervención en una población creciente.

\section{Materiales y métodos}

Se trata de un estudio analítico de cohorte cuya población de estudio y unidad de análisis fueron pacientes ancianos sometidos a cirugía cardiovascular. La muestra se calculó con base en la prevalencia de fragilidad que se ha observado en estudios previos y el riesgo de complicaciones asociadas a esta condición. Se consideró un poder del $90 \%$, tomando en cuenta una muestra mínima de 60 sujetos. Los criterios de elección fueron edad igual o mayor a 60 años, pacientes elegidos a cirugía por el grupo quirúrgico de la institución (cirugía de revascularización miocárdica, reemplazo y reparo valvular vía esternotomía). Se excluyeron aquellos que necesitaran procedimiento de emergencia, que estuvieran hospitalizados en unidad de cuidado intermedio o intensivo, que tuvieran condición neuropsiquiátrica o emocional severa que impidiera la cooperación para realización de las mediciones o aquellos que no firmaran o desistieran del consentimiento informado. El estudio fue aprobado por el comité de bioética de la Universidad Tecnológica de Pereira y fue clasificado como una investigación de riesgo mínimo según la resolución colombiana número 008430 de 1993.

Fueron evaluados a través de los criterios del índice de fragilidad de Fried $^{9}$ y las pruebas de medición se realizaron entre 1 a 5 días previos a la cirugía de acuerdo con la estabilidad clínica del paciente. Los criterios para definir fragilidad fueron pérdida de peso, agotamiento, actividad física, velocidad de la marcha y fuerza de agarre. La pérdida de peso se definió como la pérdida de peso involuntaria de más de 10 libras en el año anterior a la cirugía. El agotamiento se determinó a partir de un autoinforme en dos ítems tomados del Center for Epidemiologic Studies Depression Scale (CES-D) ${ }^{26}$. La actividad física se calculó a partir de la escala de ejercicio para medir la función en las actividades avanzadas de la vida diaria (AADL). Los valores de fuerza de agarre se obtuvieron mediante el uso de un dinamómetro marca Takei modelo Smedley Hand Dynamometer III, que mide la fuerza de agarre en kilogramos/fuerza $(\mathrm{kg} / \mathrm{f})$ y se consideraron como criterios de debilidad valores inferiores a $26 \mathrm{~kg} / \mathrm{f}$ para los hombres y $16 \mathrm{~kg} / \mathrm{f}$ para las mujeres; estos puntos de corte se basaron en los criterios de la Foundation for the National Institutes of Health $(\mathrm{FNIH})^{27}$. La velocidad de la marcha medida en metros/segundos es una medida que está validada en la literatura para realizarse en una distancia de 3, 5 o 10 metros $^{28}$, en cuyo caso la distancia en 5 metros es más utilizada. Estudios previos han evidenciado que la distancia no tiene un impacto estadísticamente significativo en la velocidad, de modo que se puede utilizar cualquiera de estas ${ }^{29}$. Por la enfermedad cardiovascular de los pacientes y para disminuir la probabilidad del desarrollo de síntomas cardiopulmonares durante la misma, en este estudio se utilizó la medición en 3 metros. Durante la prueba de la marcha se le pidió a los pacientes marchar de manera habitual desde una posición de pie y se evaluaba el tiempo en recorrer los 3 metros; en todos los pacientes se hicieron dos mediciones de la marcha y se tomaba el registro con mayor velocidad. La lentitud se definió como una velocidad inferior a $0,8 \mathrm{~m} / \mathrm{s}$ y este punto de corte se utilizó con base en el Estudio Internacional de Movilidad en el Envejecimiento $\left(\right.$ IMIAS) ${ }^{27}$.

Los datos sobre las características preoperatorias, clínicas y demográficas de los pacientes, así como los resultados quirúrgicos se obtuvieron de la historia clínica y de una encuesta hecha antes de la cirugía. Se realizó seguimiento a un mes luego de la cirugía a partir de contacto telefónico. El resultado primario se definió como un compuesto de cualquier causa de mortalidad (<30 días) y los siguientes: infarto de miocardio postoperatorio, reintervención (cualquier causa) y rehospitalización por infarto de miocardio o 
insuficiencia cardíaca. El resultado secundario incluyó los siguientes eventos: accidente cerebrovascular (ACV), insuficiencia renal postoperatoria, ventilación prolongada ( $>24$ horas), uso prolongado de vasoactivos (> 24 horas), neumonía nosocomial, infección del tracto urinario, fibrilación auricular posoperatoria, días en la unidad de cuidados intensivos y días en el hospital. Además de la fragilidad, también se evaluó en todos los pacientes el índice de masa corporal (IMC), puntaje EuroSCORE II, Minimental test e índice de comorbilidad de Charlson.

Se realizó un análisis univariado para las variables sociodemográficas y clínicas, utilizando estadísticas descriptivas de acuerdo con el tipo de variable, para las nominales, tablas de distribución de frecuencias, porcentajes y rango. Para las variables continuas, además de las anteriores, se utilizaron medidas de tendencia central (promedio, mediana) y de dispersión (desviación estándar y rango intercuartílico). Para el análisis bivariado se usó el estadístico chi cuadrado $\left(X^{2}\right)$ para las variables categóricas y los test de $T$ de Student y la $U$ de Mann-Whitney para variables continuas según el supuesto de normalidad. Se determinó como nivel de significación estadística una $\mathrm{p}<0,05$. A partir de las variables que fueron estadísticamente significativas en el análisis bivariado se realizó el cálculo del riesgo relativo (RR) con el intervalo de confianza al 95\% (IC95\%) para aquellos desenlaces que se consideraron de interés. También se realizó un análisis de tiempo a evento con gráficas de supervivencia a partir del estimador de Kaplan-Meier.

Finalmente se hizo una prueba de riesgos proporcionales para verificar el mejor modelo de regresión a utilizar; el modelo utilizado fue una regresión de Cox (riesgo proporcional $p=0,39$ ) teniendo en cuenta las variables que fueran estadísticamente significativas y aquellas que tuvieran plausibilidad clínica y se realizaron múltiples modelos para finalmente seleccionar el más parsimonioso que permitiera una predicción más precisa y válida de la respuesta evaluada. Para facilitar el análisis y la construcción de los modelos de regresión de Cox se recategorizaron algunas variables continuas como categóricas con base en las definiciones encontradas en la literatura. El Mini mental Test se clasificó en aquellos con y sin deterioro cognoscitivo a partir del puntaje obtenido y su ajuste por nivel educativo (21 puntos para sujetos con más de 6 años de estudio, 24 para sujetos con 7-12 años de estudio y 27 para sujetos con menos de 12 años). De acuerdo con estudios colombianos ${ }^{25,30}$, el puntaje de EuroSCORE II fue definido en aquellos que tuvieran riesgo bajo (puntaje $<2$ ) y aquellos con riesgo intermedio 0 alto (puntaje $>2$ ) ${ }^{31}$. Debido a que todos los pacientes tuvieron algún grado de comorbilidad, el índice de comorbilidad de Charlson se clasificó de acuerdo con la descripción inicial de este puntaje ${ }^{23}$ en aquellos que tenían un índice muy alto cuando su puntaje fuera mayor a 3 vs. los que no; respecto al número de medicamentos se dividió en aquellos que cumplieran criterios de polifarmacia con base en la definición del uso de 5 o más medicamentos ${ }^{18}$. En cuanto a los días en la UCI se consideró estancia prolongada una duración mayor o igual a 3 días (72 horas).

Los análisis se hicieron a través del software SPSS versión 20.0 (SPSS Inc., Chicago, IL, USA ${ }^{\circledast}$ ) para el cálculo del riesgo, en tanto que para la regresión de Cox se utilizó el programa Stata (StataCorp ${ }^{\circledR}$ ).

\section{Resultados}

En este estudio se evaluaron 71 pacientes entre julio de 2016 y octubre de 2017. El análisis final incluyó 66 pacientes, ya que 5 fueron excluidos por cancelación de la cirugía a último momento. Todos los pacientes firmaron el consentimiento informado y no se registraron complicaciones durante la entrevista, las mediciones de velocidad de la marcha y la dinamometría. La prevalencia de fragilidad en este estudio fue de $31,8 \%(21 / 66)$, mientras que los no frágiles fueron el $68,2 \%(45 / 66)$, los cuales se distribuyeron en vigorosos con un $22,7 \%$ (15/66) y prefrágiles con un 45,5\% (30/66) (fig. 1).

Las características sociodemográficas se describen en la tabla 1. La mayor parte de los pacientes era de sexo masculino tanto en población frágil como no frágil. El porcentaje de mujeres para pacientes frágiles y no frágiles fue de $38,1 \%$ y $28,9 \%$ respectivamente, sin diferencia estadística $(p 0,45)$. Los pacientes frágiles tenían una edad mayor que los no frágiles y la diferencia fue estadísticamente significativa (72 $\pm 6,0$ vs. $68,9 \pm 5,9$, p 0,05). Igualmente, se encontró una diferencia estadísticamente significativa en las siguientes variables: diabetes mellitus (frágiles $=52,4 \%$ vs. no frágiles $=24,3 \%$, p 0,02), enfermedad renal crónica (frágiles $=33,3 \%$ vs. no frágiles $=6,7 \%$, p 0,005), minimental test (frágiles $=24( \pm 4,7)$ vs. no frágiles $=26( \pm 2,1), p$ 0,01 , hemoglobina prequirúrgica (frágiles $=12,5( \pm 1,6) \mathrm{vs}$. no frágiles $=13,3( \pm 1,6)$, p 0,05), puntaje de EuroSCORE II (frágiles =4,8 $( \pm 6,4)$ vs. no frágiles =2,3 $( \pm 1,8), p 0,01)$; también hubo diferencias en el número de medicamentos, comorbilidades e índice de comorbilidad de Charlson (tabla $1)$.

De las 5 variables que definen la fragilidad (pérdida de peso, debilidad o agotamiento, fuerza de agarre, velocidad de la marcha y actividad física) se encontró diferencia estadísticamente significativa en cada una de ellas, como era de esperarse (tabla 2).

En la tabla 3 se describen las características quirúrgicas de los pacientes subdivididos por grupos. El tipo de cirugía más frecuente fue la revascularización en ambos grupos, seguido por la cirugía valvular y por último la cirugía mixta. No hubo diferencias estadísticamente significativas en los tipos de procedimientos entre pacientes frágiles vs. no frágiles ( $p$,97). Respecto al subgrupo de valvulopatías, la válvula que más se intervino fue la aórtica $(n=16)$, y en su mayoría fueron pacientes no frágiles [no frágiles $=11(69 \%)$ vs. frágiles $=5(31 \%)]$. La intervención de la válvula mitral se realizó en 10 pacientes, 3 fueron frágiles $(30 \%)$ y los 7 restantes (70\%) no lo fueron. La media del tiempo de bomba medida en minutos para los pacientes frágiles, fue mayor que la media de los pacientes no frágiles con una diferencia estadísticamente significativa (frágiles $=105,9 \pm 58,7 \mathrm{~min}$ vs. no frágiles $=85,6 \pm 22,9 \mathrm{~min}$, p 0,04). No se encontraron diferencias en el tiempo de pinzamiento de la aorta (frágiles $=69,7 \pm 33,2$ min vs. no frágiles $=85,6 \pm 21,1 \mathrm{~min}, \mathrm{p} \mathrm{0,46)}$.

Se efectuó inicialmente una medición respecto a los resultados postoperatorios. Se encontró que los pacientes frágiles tenían mayores resultados adversos en el resultado primario combinado (frágiles $=61,9 \%$ vs. no frágiles $=34,4 \%$, p 0,006). El resultado del punto primario fue dependiente de la mortalidad ( $\mathrm{p} 0,001)$ y las variables de IAM postquirúrgico 


\section{VALORACIÓN DE FRAGILIDAD}

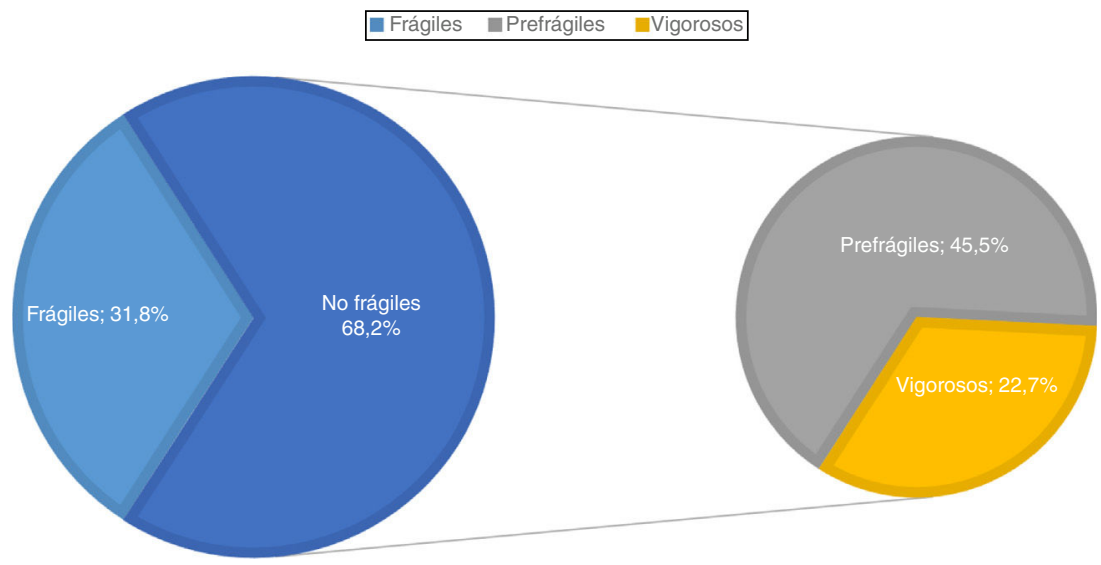

Figura 1 Prevalencia de fragilidad en población de estudio.

Tabla 1 Características sociodemográficas y clínicas en la población de estudio. Los datos se presentan en frecuencia (\%) y medias (DE)

\begin{tabular}{llll}
\hline Característica & Frágil $(\mathrm{n}=21)$ & No frágil $(\mathrm{n}=45)$ & Valor de $p$ \\
\hline Edad (años) & $72( \pm 6.0)$ & $68.9( \pm 5.9)$ & 0.05 \\
Mujeres (\%) & 38.1 & 28.9 & 0.45 \\
IMC $\left(\mathrm{kg} / \mathrm{m}^{2}\right)$ & $25.5( \pm 4.4)$ & $25.3( \pm 4.1)$ & 0.83 \\
Tabaquismo (\%) & 66.7 & 53.3 & 0.30 \\
Diabetes mellitus (\%) & 52.4 & 24.4 & 0.02 \\
Hipertensión arterial (\%) & 85.7 & 80.0 & 0.57 \\
Fibrilación auricular (\%) & 9.5 & 6.7 & 0.68 \\
Enfermedad renal crónica (\%) & 33.3 & 6.7 & 0.005 \\
IAM previo(\%) & $38.1 \%$ & $48.9 \%$ & 0.41 \\
Fracción de eyección (\%) & $49.0( \pm 12.0)$ & $52.4( \pm 9.6)$ & 0.22 \\
Minimental test & $24( \pm 4.7)$ & $26( \pm 2.1)$ & 0.01 \\
HB prequirúrgica (g/dl) & $12.5( \pm 1.6)$ & $13.3( \pm 1.6)$ & 0.05 \\
Creatinina prequirúrgica (mg/dl) & $1.0( \pm 0.32)$ & $1.1( \pm 1.1)$ & 0.67 \\
EUROSCORE II & $4.8( \pm 6.4)$ & $2.3( \pm 1.8)$ & 0.01 \\
$\mathrm{~N}^{\circ}$ Medicamentos & $4.8( \pm 2.1)$ & $3.24( \pm 2.2)$ & 0.009 \\
$\mathrm{~N}^{\circ}$ comorbilidades & $3.1( \pm 1.1)$ & $2.0( \pm 1.0)$ & 0.001 \\
Índice de Charlson & $5.19( \pm 1.5)$ & $4.2( \pm 1.5)$ & 0.01 \\
\hline
\end{tabular}

* Porcentaje de mujeres dentro del total de pacientes frágiles $(8 / 21)$ y no frágiles $(13 / 45)$

Tabla 2 Características definitorias de fragilidad en los dos grupos. Los datos se presentan en frecuencia (\%) y medias (DE)

\begin{tabular}{llll}
\hline Característica & Frágil $(\mathrm{n}=21)$ & No frágil $(\mathrm{n}=45)$ & Valor de $p$ \\
\hline Pérdida de peso (\%) & 52.4 & 17.8 & 0.04 \\
Debilidad o agotamiento $(\%)$ & 42.9 & 13.4 & 0.03 \\
Fuerza de agarre $(\mathrm{kg} / \mathrm{f})$ & $21.1( \pm 6.6)$ & $28.1( \pm 7.5)$ & 0.001 \\
Velocidad de la marcha $(\mathrm{m} / \mathrm{s})$ & $0.67( \pm 0.23)$ & $0.99( \pm 0.26)$ & 0.00 \\
Actividad física $(\%)$ & 80.9 & 45.7 & 0.04 \\
\hline
\end{tabular}

Tabla 3 Tipos de cirugía realizada en los subgrupos estudiados. No hubo diferencias estadísticamente significativas en los tipos de procedimientos ( $\mathrm{p}$ 0.97)

\begin{tabular}{llll}
\hline Característica & Frágil $(\mathrm{n}=21)$ & No frágil $(\mathrm{n}=45)$ & Total $(\mathrm{n}=66)$ \\
\hline Revascularización & $61.9 \%(13)$ & $60.0 \%(27)$ & $60.6 \%(40)$ \\
Valvular & $28.6 \%(6)$ & $28.9 \%(13)$ & $28.8 \%(19)$ \\
Mixta & $9.5 \%(2)$ & $11.1 \%(5)$ & $10.6 \%(7)$ \\
\hline
\end{tabular}


Tabla 4 Resultados de los desenlaces quirúrgicos. Se describen los resultados en frecuencia (\%) y entre paréntesis el número (n) de pacientes

\begin{tabular}{lllll}
\hline Variable $\%$ & Frágiles $(\mathrm{n}=21)$ & No frágiles $(\mathrm{n}=45)$ & Total $(\mathrm{n}=66)$ & Valor de $\mathrm{p}$ \\
\hline Resultado primario & $61.9(13)$ & $24.4(11)$ & $34.4(24)$ & 0.006 \\
Mortalidad total & $38.1(8)$ & $4.4(2)$ & $15.2(10)$ & 0.001 \\
IAM postquirúrgico & $8.9(4)$ & $19.0(4)$ & $12.1(8)$ & $16.7(11)$ \\
Reintervención & $19.0(4)$ & $15.6(7)$ & $9.1(6)$ & 0.23 \\
Rehospitalización cardiovascular & $14.3(3)$ & $6.7(3)$ & $37.9(25)$ & 0.72 \\
Uso prolongado de vasoactivos & $66.7(14)$ & $24.4(11)$ & $31.8(21)$ & 0.31 \\
FA postoperatoria & $42.9(9)$ & $26.7(12)$ & $25.8(17)$ & 0.25 \\
Ventilación prolongada & $52.4(11)$ & $13.3(6)$ & $10.7(11)$ & 0.001 \\
Rehospitalización (todas las causas) & $23.8(5)$ & $13.3(6)$ & $13.6(9)$ & 0.30 \\
Mortalidad cardiovascular & $38.1(8)$ & $2.2(1)$ & $12.1(8)$ & 0.000 \\
Mortalidad hospitalaria & $33.3(7)$ & $2.2(1)$ & $7.6(5)$ & 0.001 \\
Infección urinaria & $14.3(3)$ & $4.4(2)$ & $6.1(4)$ & 0.15 \\
Infección sitio operatorio & $14.3(3)$ & $2.2(1)$ & $3.0(2)$ & 0.04 \\
Neumonía & $4.8(1)$ & $2.2(1)$ & $1.5(1)$ & 0.57 \\
Enfermedad cerebrovascular & $0(0)$ & $2.2(1)$ & 0.49 \\
\hline
\end{tabular}

(p 0,23), reintervención por cualquier causa $(\mathrm{p} 0,72)$ y rehospitalización cardiovascular ( $\mathrm{p} 0,31)$ no mostraron diferencias estadísticamente significativas.

Respecto a los resultados secundarios se encontró que los pacientes frágiles tuvieron mayor incidencia de uso prolongado de vasoactivos (frágiles $=66.7 \%$ vs. no frágiles $=24.4 \%$, p 0.001 ), ventilación prolongada (frágiles $=52.4 \%$ vs. no frágiles $=13.3 \%$, p 0.001), mortalidad cardiovascular (frágiles $=38.1 \%$ vs. no frágiles $=2.2 \%$, p 0.000 ), mortalidad hospitalaria (frágiles $=33.3 \%$ vs. no frágiles $=2.2 \%, p 0.001$ ) e infección del sitio operatorio (frágiles $=14.3 \%$ vs. no frágiles $=6.1 \%$, p 0.04). Para el análisis de la estancia en la Unidad de Cuidado Intensivo se excluyeron los pacientes fallecidos en el hospital $(n=6)$ ya que una mortalidad temprana podría interferir con los resultados; en consecuencia, se encontró que los pacientes frágiles tenían una estancia más prolongada (frágiles =5.5 $(\mathrm{RI}=5.0)$ vs. no frágiles=3.0 $(\mathrm{RI}=3.0)$, p 0.006). Otras variables evaluadas no tuvieron diferencias estadísticas (tabla 4).

Se realizó un análisis del riesgo relativo (RR) otorgado de la fragilidad para las complicaciones postoperatorias. Sin duda el hallazgo más importante fue la asociación fuerte de la mortalidad con la fragilidad (RR 8.5, IC95\% 1.9-36.9). Otras condiciones que incrementaron el riesgo de mortalidad fueron la ventilación prolongada (RR 11.5, IC95\% 12.7-49.0) y el uso prolongado de vasoactivos (RR 3.8, IC95\% $1.0-13.4)$. No se encontró riesgo con otras variables adversas como infarto agudo de miocardio previo a la cirugía (RR 1.8, IC95\% 0.5-5.7), EuroSCORE II alto (RR 3.13, IC95\% 0.713.6), edad mayor a 75 años (RR 1.4, IC95\% 0.4-4.9), índice de comorbilidad de Charlson alto (RR 2.6, IC95\% 0.3-19.2), deterioro cognitivo (RR 0.32, IC95\% 0.04-2.34), FEVI baja (<50\%) (RR 1.4, IC95\% 0.4-4.5), infección del sitio operatorio (RR 0.7, IC95\% 0.2-2.2) y estancia prolongada en UCI (RR 0.7, IC95\% 0.2-2.2).

Adicionalmente, se realizó el cálculo del RR para cada variable de fragilidad y la condición de fragilidad la cual se ilustra en la tabla 5 . Como se puede observar ninguna variable por sí sola tuvo el incremento del riesgo; por
Tabla 5 Riesgo relativo de cada una de las variables de la fragilidad

\begin{tabular}{ll}
\hline Variable & Riesgo relativo RR (IC 95\%) \\
\hline Pérdida de peso & $1.6(0.3-3.6)$ \\
Debilidad o agotamiento & $1.2(0.8-1.6)$ \\
Marcha lenta & $3.1(0.89-11.1)$ \\
Dinapenia (baja fuerza de agarre) & $2.6(0.8-8.1)$ \\
Inactividad física & $1.1(0.9-1.4)$ \\
Fragilidad (3 o más criterios) & $8.5(1.9-36.9)$ \\
\hline
\end{tabular}

el contrario, la suma de las variables, es decir, el tener fragilidad sí mostró un riesgo asociado.

También se realizó una curva de Kaplan-Meier en la cual se grafica la estimación de la probabilidad de supervivencia de los pacientes intervenidos de cirugía cardiaca en un intervalo de tiempo de 30 días según la condición de fragilidad. La supervivencia estimada en los pacientes frágiles fue de 22.6 (límite inferior: 18.0 días, límite superior: 27.1 días), mientras que los en los no frágiles fue de 28.7 días (límite inferior: 18.0 días, límite superior: 27.1 días), con una diferencia estadísticamente significativa (p 0.00) (fig. 2).

Finalmente se construyó un modelo de regresión de Cox a partir de variables que mostraron significancia estadística en el análisis bivariado y aquellas con plausibilidad clínica con el fin de analizar la supervivencia de los pacientes de la cohorte. Se utilizaron varios modelos de predicción y se seleccionó el más parsimonioso. Las variables asociadas con la mortalidad fueron la polifarmacia, la fragilidad y un puntaje EuroSCORE II alto. El modelo tuvo significancia estadística (p 0.000) (tabla 6).

\section{Discusión}

Este es el primer estudio en Colombia diseñado para evaluar las implicaciones de la fragilidad en el anciano sometido a cirugía cardiaca. Se encontró que la fragilidad es una 


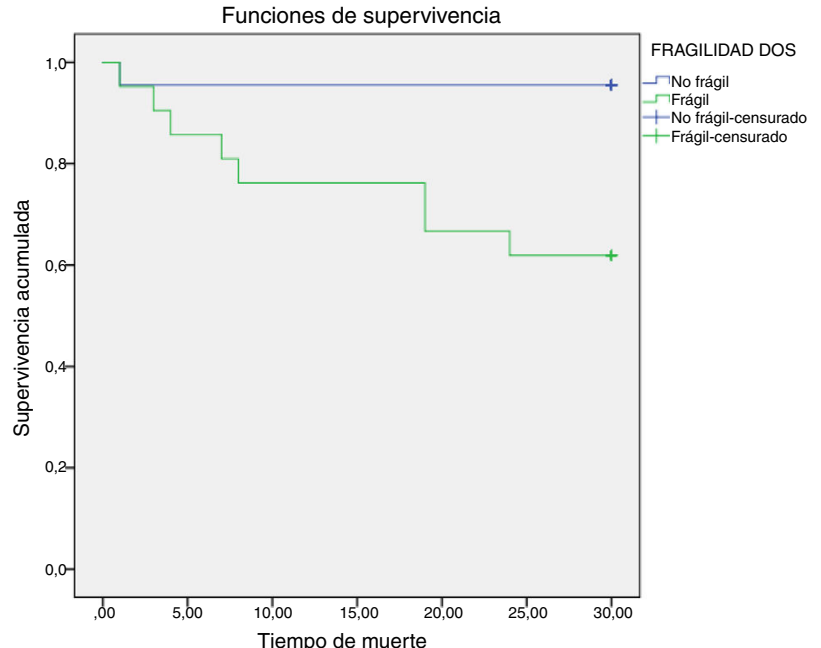

Figura 2 Análisis de Kaplan-Meier con la supervivencia según fragilidad. Tiempo de muerte establecido en un periodo comprendido de 0-30 días.

condición frecuente y se comporta como un factor de riesgo independiente de desenlaces adversos como mortalidad, ventilación prolongada e infección del sitio operatorio. El incremento del riesgo de presentar un resultado primario combinado dependió fundamentalmente de la mortalidad temprana de los pacientes (evaluada 30 días). Otras condiciones prequirúrgicas que impactaron en la mortalidad fueron, como era de esperarse, tener un EuroSCORE II elevado (puntaje $>2$ ) y adicionalmente tener polifarmacia, una condición muy frecuente en los pacientes geriátricos quirúrgicos ${ }^{19}$ pero poco estudiada en relación con la cirugía cardiaca, lo cual resulta ser un dato novedoso de este estudio.

La prevalencia de fragilidad en este estudio fue del $31.5 \%$, porcentaje que se encuentra en las prevalencias esperadas para la población anciana con enfermedad cardiovascular, la cual varía entre el $20-40 \%{ }^{29}$. No obstante, la prevalencia de fragilidad debe interpretarse de manera crítica ya que depende de múltiples condiciones como el tipo de población estudiada, el contexto clínico donde se realizó la medición y el tipo de herramienta utilizada ${ }^{37,38}$. Por ejemplo, si se comparan los resultados con estudios que evaluaron la fragilidad en ancianos colombianos de la comunidad ${ }^{26,32}$ la prevalencia en este estudio fue mayor ( 31.5 vs. $12.1 \%$ ). Otros estudios como el de Ekerstad et al. ${ }^{79}$ que han evaluado la prevalencia de fragilidad en ancianos enfermos desde el punto de vista cardiovascular (síndrome coronario agudo), han reportado una prevalencia mucho mayor (48.5\%). Por otro lado, si se comparan estos resultados con los estudios que han evaluado la fragilidad en el contexto prequirúrgico de la cirugía cardiaca (como en este estudio) se observa una importante heterogeneidad (tabla 7) puesto que la población de este estudio está en un rango intermedio de prevalencia; sin embargo, al compararlo con el estudio de Afilalo y Niv ad, que utilizaron también el índice de Fried la prevalencia fue mayor (20 y $23 \%$ respectivamente vs. $31.5 \%$ para este estudio) lo cual indica que en esta población se operó un mayor porcentaje de pacientes frágiles.

Un número creciente de publicaciones han evaluado el impacto de la fragilidad en el contexto quirúrgico ${ }^{4,33-36}$. Para este estudio la fragilidad incrementaba el riesgo de mortalidad en 8.5 veces (RR 8.5, IC95\% 1.9-36.9). Cuando se comparó este estudio con otros que evaluaron la fragilidad en el contexto de cirugía cardiaca se hallaron algunas diferencias (tabla 8), por ejemplo, una muy alta mortalidad en los pacientes frágiles (este estudio: $38.1 \%$ vs. Lee et al. ${ }^{13}$ : $14.7 \%$, Afilalo et al. ${ }^{4}: 1.8-2.6 \%$, Niv ad et al. ${ }^{39}: 2.6 \%$ ). A pesar de lo anterior, un dato interesante es que la incidencia de mortalidad en algunos estudios como el de Sunderman et al. ${ }^{34}$ dependió del grado de fragilidad asignado, por ejemplo, si se consideraba que el paciente tenía un grado leve la mortalidad fue de $5.5 \%$, en cambio si se consideraba severa la mortalidad se incrementaba hasta el $21 \%$; este tipo de resultados llevó a plantearse si una de las explicaciones para que la población de este estudio tuviera mayor mortalidad estuviera determinado por un grado mayor de fragilidad y se evidenció que los resultados en la velocidad de la marcha (uno de los ítem de la fragilidad) estaban muy por debajo de otras poblaciones. En el estudio de Afilalo et al. ${ }^{4}$ la velocidad de la marcha fue de $0.94 \mathrm{~m} / \mathrm{s}$ mientras que en este estudio la medida de la velocidad de la marcha fue 0.86 $\mathrm{m} / \mathrm{s}$; adicionalmente, la media de los pacientes frágiles fue mucho más baja $0.67 \mathrm{~m} / \mathrm{s}$. Como se demostró en el estudio de Afilalo et al. ${ }^{4}$ existe una relación inversamente proporcional entre la velocidad de la marcha y la mortalidad (el OR para mortalidad se incrementa 1.03 por cada reducción de $0.1 \mathrm{~m} / \mathrm{s}$ ) lo cual puede interpretarse como que al ser más lentos los pacientes de este estudio era de esperarse una mayor mortalidad.

Para este estudio el EuroSCORE II subestimaba el riesgo de mortalidad; el riesgo medido por esta escala fue mucho menor al observado $(2.24 \%$ vs. $15.2 \%)$, a pesar de que es

Tabla 6 Modelo de regresión de Cox para mortalidad

\begin{tabular}{|c|c|c|c|c|c|c|}
\hline \multirow[b]{3}{*}{ Variable } & \multicolumn{6}{|c|}{ Modelo de regresión de Cox: mortalidad } \\
\hline & \multicolumn{5}{|c|}{ Log Likelihood $=-28.12$, LR $\mathrm{chi}^{2}=20.25$, Prob $>\mathrm{chi}^{2}=0.0000$} & \\
\hline & $\mathrm{HR}$ & $\mathrm{EE}$ & Z & $P>|z|$ & IC $95 \%$ & \\
\hline IAM postquirúrgico & 11.9 & 9.36 & 3.17 & 0.002 & 2.5 & 55.4 \\
\hline Polifarmacia & 9.27 & 8.23 & 2.51 & 0.012 & 1.6 & 52.8 \\
\hline Fragilidad & 5.49 & 4.62 & 2.03 & 0.043 & 1.0 & 28.5 \\
\hline EuroSCORE II & 1.17 & 0.05 & 3.16 & 0.002 & 1.0 & 1.29 \\
\hline
\end{tabular}

HR: hazard ratio, EE: error estándar, IC: intervalo de confianza, IAM: infarto agudo de miocardio. 
Tabla 7 Comparación entre la prevalencia de fragilidad en distintos estudios

\begin{tabular}{|c|c|c|c|c|}
\hline Autor (año) & Población y edad & $\mathrm{n}$ & $\begin{array}{l}\text { Definición de } \\
\text { fragilidad }\end{array}$ & $\begin{array}{l}\text { Prevalencia } \\
\text { fragilidad }\end{array}$ \\
\hline $\begin{array}{l}\text { Lee et al.(13) ^ } \\
(2010)\end{array}$ & Cirugía cardiaca & 3826 & $\begin{array}{l}\text { Índice de Katz } \\
\text { Deambulación } \\
\text { Demencia }\end{array}$ & $4.1 \%$ \\
\hline $\begin{array}{l}\text { Sundermann } \\
\text { et al. }{ }^{34}(2011)\end{array}$ & $\begin{array}{l}\text { Cirugía cardiaca } \\
\geq 74 \text { años }\end{array}$ & 400 & CAF simplificado & $50.1 \% * *$ \\
\hline $\begin{array}{l}\text { Afilalo et al. }{ }^{4} \\
(2012)\end{array}$ & $\begin{array}{l}\text { Cirugía cardiaca } \\
\geq 70 \text { años }\end{array}$ & 152 & $\begin{array}{l}\text { Índice CHS } \\
\text { Índice CHS } \\
\text { modificado } \\
\text { Índice MSSA } \\
\text { Velocidad de } \\
\text { marcha } 5 \text { metros }\end{array}$ & $20-46 \%$ * \\
\hline $\begin{array}{l}\text { Afilalo et al. }{ }^{33} \\
(2016)\end{array}$ & $\begin{array}{l}\text { Cirugía cardiaca } \\
\geq 60 \text { años }\end{array}$ & 15.171 & $\begin{array}{l}\text { Velocidad de } \\
\text { marcha en } 5 \\
\text { metros }\end{array}$ & $30.2 \%$ \\
\hline $\begin{array}{l}\text { Niv ad et al. }{ }^{39} \\
(2016)\end{array}$ & $\begin{array}{l}\text { Cirugía cardiaca } \\
\geq 65 \text { años }\end{array}$ & 166 & Índice CHS & $23 \%$ \\
\hline $\begin{array}{l}\text { Este estudio } \\
(2018)\end{array}$ & $\begin{array}{l}\text { Cirugía cardiaca } \\
\geq 60 \text { años }\end{array}$ & 66 & Índice CHS & $31.5 \%$ \\
\hline
\end{tabular}


Tabla 8 Comparación de mortalidad entre distintos estudios según la fragilidad

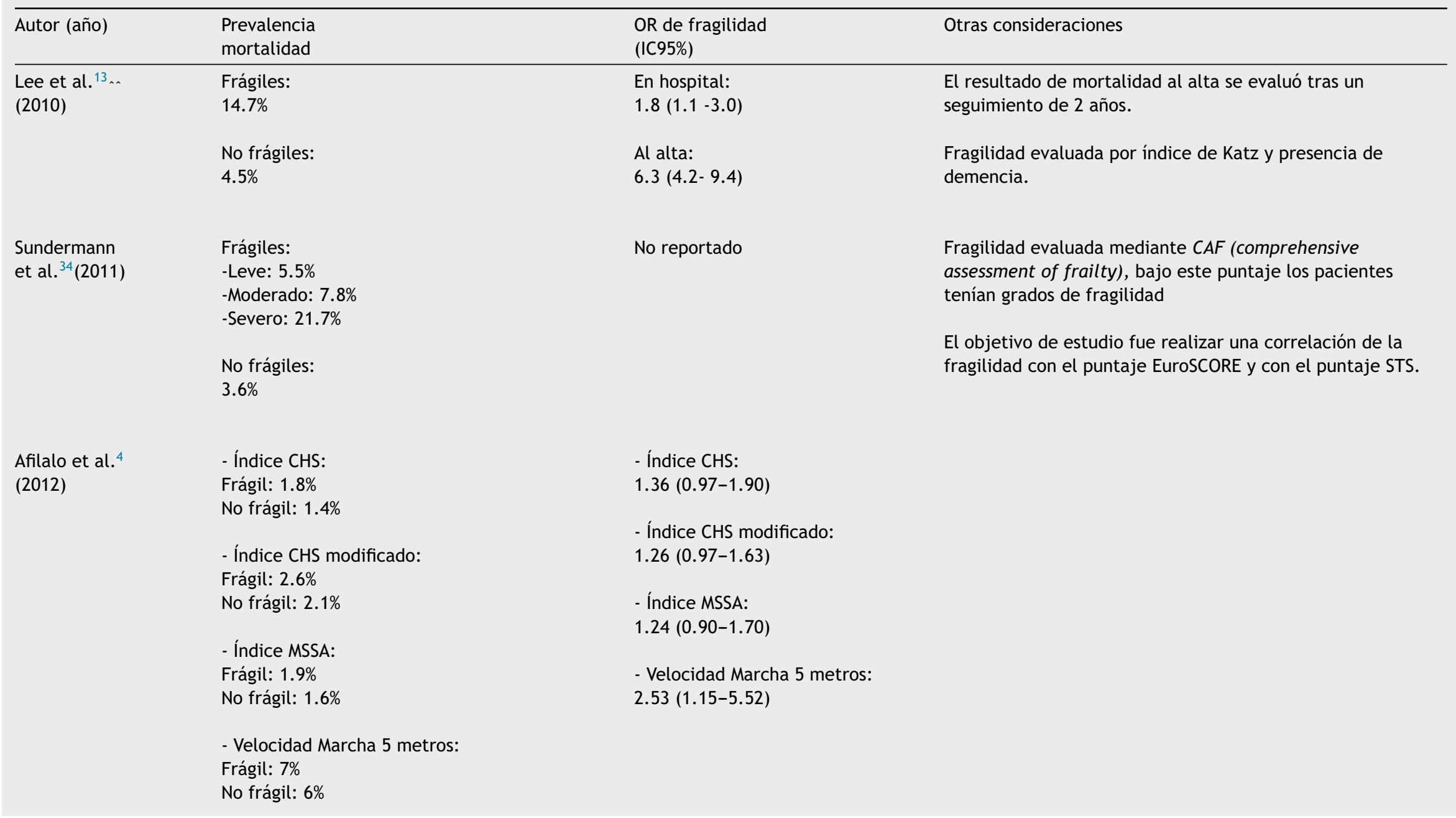




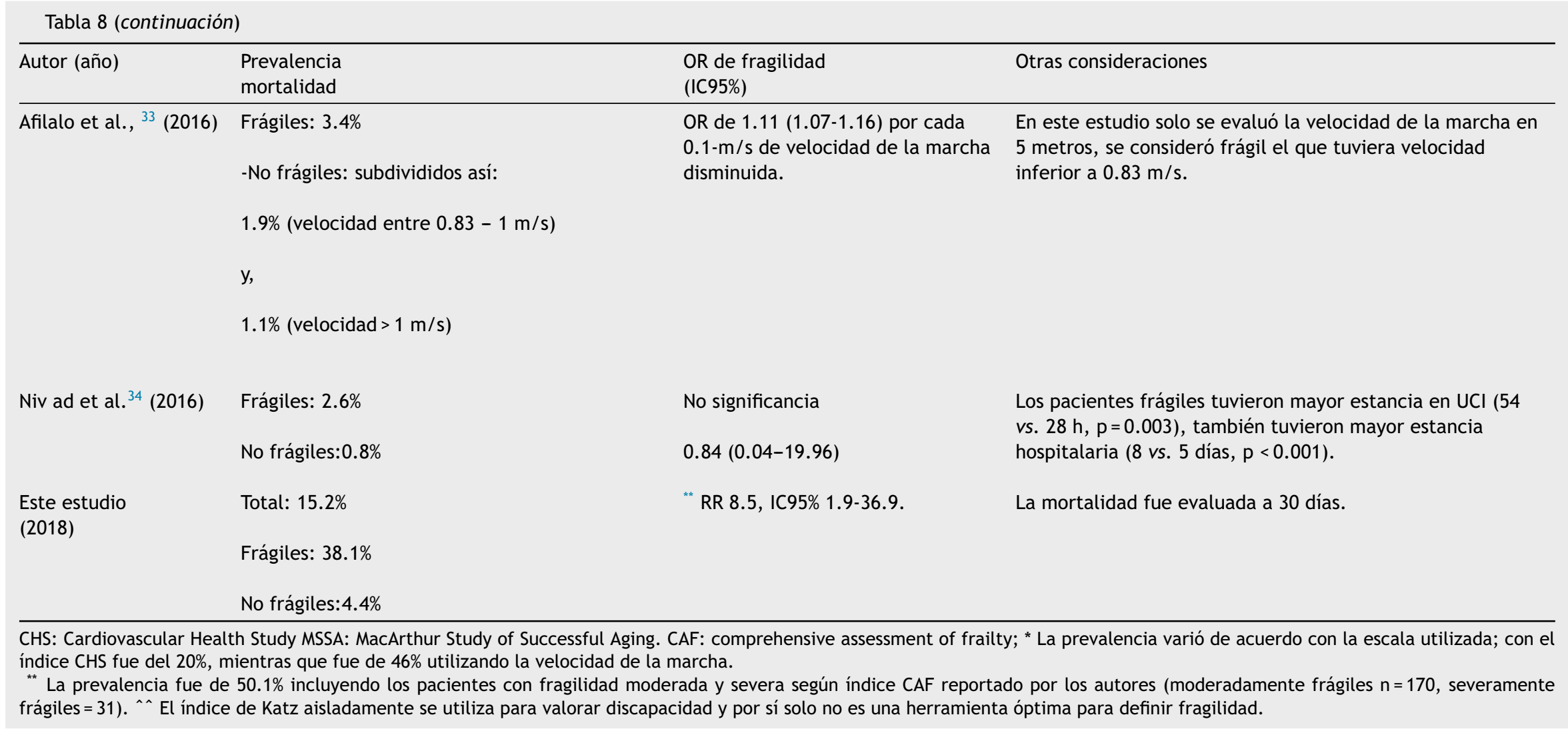


la escala de amplio uso en el contexto prequirúrgico de la cirugía cardiaca, tener un puntaje alto tuvo un HR más bajo que la fragilidad (HR 1.17 IC95\% 1.0-1.29 vs. HR 5.49 IC95\% 1.0-28.5). Consideramos que para mejorar la predicción del riesgo de mortalidad en población anciana se deben tener en cuenta otras variables prequirúrgicas como la polifarmacia y la fragilidad. Estudios como el de Sunderman et al. ${ }^{34}$ han logrado mejorar la predicción de desenlaces adversos de cirugía cardiaca al añadir al modelo EuroSCORE II y al modelo STS variables que valoran la fragilidad. Afilalo et al. ${ }^{4}$ también lograron mejorar el área bajo la curva $(A B C)$ del puntaje STS cuando añadían variables de fragilidad (ABC del STS: 0.68-0.72 vs. STS + fragilidad: $0.73-0.76)$. Lo anterior sustenta que en pacientes ancianos se debe hacer un esfuerzo por realizar medición objetiva de la fragilidad que permita refinar la predicción de los desenlaces adversos.

Estudios previos han mostrado que operar a un paciente frágil es un $30 \%$ más costoso que operar a un paciente no frágil $^{40}$. Estos datos llevan a plantear la necesidad de crear grupos multidisciplinarios donde se otorgue un papel más importante a la fragilidad, no solo para mejorar los resultados en salud, sino también para optimizar los costos en salud que acarrea esta condición.

\section{Limitaciones}

Se trata de un estudio unicéntrico lo cual impide la extrapolación de los resultados a otros centros de cirugía cardiaca con más experiencia; no obstante, se llevó a cabo en una institución que tiene influencia en toda la región del eje cafetero. Este tipo de limitación también motiva a extender este tipo de cohortes en otros lugares. Así mismo, se trata de una muestra pequeña si se la compara con otros estudios, hechi pudo implicar que la velocidad de la marcha no tuviera la potencia estadística para ser por sí sola una condición que permitiera definir un riesgo, como lo han demostrado otros estudios. Adicionalmente, se excluyeron pacientes que se encontraban en la unidad de cuidado intermedio o intensivo, lo cual impide que los resultados sean interpretables en población críticamente enferma. Consideramos que en este tipo de población se debe utilizar otro tipo de mediciones ya que la medición de la marcha o de la fuerza de agarre tienen limitaciones operativas. Finalmente, debido a que se excluyeron pacientes con cirugía emergente, estos resultados tampoco pueden ser extrapolados a esa población de pacientes.

\section{Conclusión}

La fragilidad medida por el fenotipo de Fried incrementó los resultados adversos en ancianos sometidos a cirugía cardiovascular, específicamente mortalidad, tiempo prolongado de uso de vasoactivos, ventilación mecánica prolongada e infección del sitio operatorio. Se recomienda incluir la medición de la fragilidad en el protocolo prequirúgico del paciente anciano.

\section{Financiación}

Ninguna.

\section{Conflictos de intereses}

Ninguno.

\section{Bibliografía}

1. Nicolini F, Agostinelli A, Vezzani A, Manca T, Benassi F, Molardi A, et al. The evolution of cardiovascular surgery in elderly patient: a review of current options and outcomes. Biomed Res Int [Internet]. 2014;2014:736298. Disponible en: http: / / www.pubmedcentral.nih.gov/articlerender.fcgi?artid = 4000933\&tool = pmcentrez\&rendertype $=$ Abstract .

2. Singh $M$, Stewart R, White $H$. Importance of frailty in patients with cardiovascular disease. Eur Heart J [Internet]. 2014;35:1726-31, http://dx.doi.org/10.1093/ eurheartj/ehu197.

3. Makary MA, Segev DL, Pronovost PJ, Syin D, Bandeen-Roche $\mathrm{K}$, Patel P, et al. Frailty as a Predictor of Surgical Outcomes in Older Patients. J Am Coll Surg [Internet]. 2010;210:901-8, http://dx.doi.org/10.1016/j.jamcollsurg.2010.01.028.

4. Afilalo J, Mottillo S, Eisenberg MJ, Alexander KP, Noiseux N, Perrault LP, et al. Addition of frailty and disability to cardiac surgery risk scores identifies elderly patients at high risk of mortality or major morbidity. Circ Cardiovasc Qual Outcomes. 2012;5:222-8.

5. Afilalo J, Eisenberg MJ, Morin J-F, Bergman H, Monette J, Noiseux N, et al. Gait Speed as an Incremental Predictor of Mortality and Major Morbidity in Elderly Patients Undergoing Cardiac Surgery. J Am Coll Cardiol [Internet]. 2010;56:1668-76. Disponible en: http://linkinghub.elsevier.com/retrieve/pii/ S0735109710037289.

6. García-Villarreal OA. EuroSCORE II: Cómo se usa en la práctica diaria actual. Rev Mex Cardiol. 2014;25:50-1.

7. Nilsson J, Algotsson L, Höglund P, Lührs C, Brandt J. Comparison of 19 pre-operative risk stratification models in open-heart surgery. Eur Heart J. 2006;27:867-74.

8. Rodriguez-Chavez LL, Figueroa-Solano J, Munoz-Consuegra CE, Avila-Vanzzini N, Kuri-Alfaro J. [EuroSCORE underestimate the mortality risk in cardiac valve surgery of Mexican population]. Arch Cardiol Mex. 2017;87:18-25.

9. Fried LP, Tangen CM, Walston J, Newman AB, Hirsch C, Gottdiener J, et al. Frailty in older adults: evidence for a phenotype. J Gerontol A Biol Sci Med Sci. 2001;56:M146-56.

10. Neupane I, Arora RC, Rudolph JL. Cardiac surgery as a stressor and the response of the vulnerable older adult. Exp Gerontol. 2017;87 Pt B:168-74.

11. Robinson TN, Wu DS, Pointer L, Dunn CL, Cleveland JC, Moss M. Simple frailty score predicts postoperative complications across surgical specialties. Am J Surg [Internet]. 2013;206:544-50, http://dx.doi.org/10.1016/j.amjsurg.2013.03.012.

12. Yourman LC LSJSMAWEWSAK. Prognostic indices for older adults: A systematic review. JAMA J Am Med Assoc [Internet]. 2012;307:182-92. Disponible en: https://doi.org/10.1001/ jama.2011.1966\%5Cnhttp://jama.jamanetwork.com/data/ Journals/JAMA/22485/jcr15008_182_192.pdf.

13. Lee DH, Buth KJ, Martin B-J, Yip AM, Hirsch GM. Frail patients are at increased risk for mortality and prolonged institutional care after cardiac surgery. Circulation [Internet]. 2010;121:973-8. Disponible en: http://www.ncbi.nlm.nih.gov/ pubmed/20159833.

14. Amrock LG, Deiner S. The implication of frailty on preoperative risk assessment. Curr Opin Anaesthesiol [Internet]. 2014;27:330-5. Disponible en: http://www.ncbi.nlm.nih.gov/ pubmed/24566452.

15. Alexander KP, Newby LK, Armstrong PW, Cannon CP, Gibler WB, Rich MW, et al., Acute Coronary Care in the Elderly, Part II 
ST-Segment - Elevation Myocardial Infarction A Scientific Statement for Healthcare Professionals From the American Heart Association Council on Clinical Cardiology In Collaboration With the Society of Geriatric. Circulation. 2007;115:2570-89.

16. Buta BJ, Walston JD, Godino JG, Park M, Kalyani RR, Xue KB. Frailty Assessment Instruments: Identification and Systematic Characterization of the Uses and Contexts of HighlyCited Instruments. Ageing Res Rev [Internet]. 2015;26:53-61, http://dx.doi.org/10.1016/j.arr.2015.12.003.

17. Rockwood K, Song X, Macknight C, Bergman H, Hogan DB, Mcdowell I, et al. A global clinical measure of fitness and frailty in elderly people. CMAJ. 2005;173(Appendix 1):9-13.

18. Bushardt RL, Massey EB, Simpson TW, Ariail JC, Simpson KN, Polypharmacy:. Misleading, but manageable. Clin Interv Aging [Internet]. 2008;3:383-9. Disponible en: http://www.ncbi.nlm.nih.gov/pmc/articles/PMC2546482/.

19. Kennedy JM, van Rij AM, Spears GF, Pettigrew RA, Tucker IG. Polypharmacy in a general surgical unit and consequences of drug withdrawal. $\mathrm{Br} \mathrm{J}$ Clin Pharmacol [Internet]. 2000;49:353-62. Disponible en: http://www.ncbi.nlm.nih.gov/pmc/articles/PMC2014931/.

20. Härstedt M, Rogmark C, Sutton R, Melander O, Fedorowski A. Polypharmacy and adverse outcomes after hip fracture surgery. J Orthop Surg Res [Internet]. 2016;11:151. Disponible en: http://www.ncbi.nlm.nih.gov/pmc/articles/PMC5122200/.

21. Feinstein AR. The pre-therapeutic classification of co-morbidity in chronic disease. J Chronic Dis. 1970;23:455-68.

22. Rosas-Carrasco O, González-Flores E, Brito-Carrera AM, Vázquez-Valdez OE, Peschard-Sáenz E, Gutiérrez-Robledo LM, et al. Evaluación de la comorbilidad en el adulto mayor. Rev Med Inst Mex Seguro Soc [Internet]. 2011;49:153-62. Available from: http: / / www. redalyc.org/articulo. oa?id = 457745501010.

23. Charlson ME, Pompei P, Ales KL, MacKenzie CR. A new method of classifying prognostic comorbidity in longitudinal studies: Development and validation. J Chronic Dis [Internet]. 1987;40:373-83. Disponible en: http://www. sciencedirect.com/science/article/pii/0021968187901718.

24. Stroobant N, Vingerhoets G. Pre-existing cognitive impairment in candidates for cardiac surgery: an overview. Heart [Internet]. 2009;95:1820-5. Disponible en: https://heart.bmj.com/ content $/ 95 / 22 / 1820$.

25. Rosselli D, Ardila A, Pradilla G, Morillo L, Bautista L, Rey O, et al. The Mini-Mental State Examination as a selected diagnostic test for dementia: a Colombian population study. GENECO. Rev Neurol. 2000;30:428-32.

26. Radloff LS. The CES-D Scale: A Self-Report Depression Scale for Research in the General Population Appl Psychol. Meas [Internet]. 1977;1:385-401, http://dx.doi.org/ $10.1177 / 014662167700100306$.
27. de Souza Barbosa JF, Zepeda MUP, Béland F, Guralnik JM, Zunzunegui MV, Guerra RO. Clinically relevant weakness in diverse populations of older adults participating in the International Mobility in Aging Study. Age (Omaha) [Internet]. 2016;38:25. Disponible en: http://www.ncbi.nlm.nih.gov/ pmc/articles/PMC5005882/.

28. Afilalo J, Alexander KP, Mack MJ, Maurer MS, Green P, Allen LA, et al. Frailty assessment in the cardiovascular care of older adults. J Am Coll Cardiol [Internet]. 2014;63:747-62. Disponible en: http://www.ncbi.nlm.nih.gov/pubmed/24291279.

29. Graham JE, Ostir GV, Kuo Y-F, Fisher SR, Ottenbacher KJ. Relationship between test methodology and mean velocity in timed walk tests: a review. Arch Phys Med Rehabil. 2008;89:865-72.

30. Pedraza L OL, Sánchez E, Plata SJ, Montalvo C, Galvis P, Chiquillo A. Puntuaciones del MoCA y el MMSE en pacientes con deterioro cognitivo leve y demencia en una clínica de memoria en Bogotá. Acta Neurológica Colombiana. 2014;30:22-31.

31. Nashef SAM, Roques F, Sharples LD, Nilsson J, Smith C, Goldstone AR, et al. EuroSCORE II. Eur J Cardiothorac Surg. 2012;41:734-5.

32. Gomez F, Curcio CLHG. Fragilidad en ancianos colombianos. Rev Medica Sanitas. 2012;15:8-16.

33. Afilalo J, Kim S, O'Brien S, Brennan JM, Edwards FH, Mack MJ, et al. Gait speed and operative mortality in older adults following cardiac surgery. JAMA Cardiol. 2016;1:314-21.

34. Sundermann S, Dademasch A, Praetorius J, Kempfert J, Dewey T, Falk V, et al. Comprehensive assessment of frailty for elderly high-risk patients undergoing cardiac surgery. Eur J Cardiothorac Surg. 2011;39:33-7.

35. Goldfarb M, Bendayan M, Rudski LG, Morin J-F, Langlois Y, Ma F, et al. Cost of Cardiac Surgery in Frail Compared With Nonfrail Older Adults. Can J Cardiol. 2017;33:1020-6.

36. Lytwyn J, Stammers AN, Kehler DS, Jung P, Alexander B, Hiebert BM, et al. The impact of frailty on functional survival in patients 1 year after cardiac surgery. J Thorac Cardiovasc Surg. 2017;154:1990-9.

37. Beggs T, Sepehri A, Szwajcer A, Tangri N, Arora RC. Frailty and perioperative outcomes: a narrative review. Can J Anaesth. 2015;62:143-57.

38. Hodari A, Hammoud ZT, Borgi JF, Tsiouris A, Rubinfeld IS. Assessment of morbidity and mortality after esophagectomy using a modified frailty index. Ann Thorac Surg. 2013;96:1240-5.

39. Ad N, Holmes SD, Halpin L, Shuman DJ, Miller CE, Lamont D. The Effects of Frailty in Patients Undergoing Elective Cardiac Surgery. J Card Surg. 2016;31:187-94.

40. Goldfarb M, Bendayan M, Rudski LG, Morin JF, Langlois Y, Ma F, et al. Cost of cardiac surgery in frail compared with nonfrail older adults. Can J Cardiol. 2017;33:1020-6. 